\title{
What do cardiologists know about the effects of cardiovascular agents on sexual function? A survey among Dutch cardiologists. Part I
}

\author{
M. P. J. Nicolai • S. S. Liem • S. Both • R. C. M. Pelger • \\ H. Putter • M. J. Schalij • H. W. Elzevier \\ Published online: 26 September 2013 \\ (C) The Author(s) 2013. This article is published with open access at Springerlink.com
}

\begin{abstract}
Introduction Several cardiovascular agents, such as diuretics and $\beta$-blockers, can negatively affect sexual function, leading to noncompliance with therapy. Others such as angiotensin II receptor blockers (ARBs) can improve patients' sexual function.

Aims We aimed to gain insight into cardiologists' knowledge about the effects of cardiovascular drugs on sexual function and whether they take this knowledge into account when prescribing drugs.

Methods An anonymous questionnaire was mailed to 980 members of the Netherlands Society of Cardiologists (cardiologists and residents in training).

Results Almost $54 \%$ of Dutch cardiologists responded; 414 questionnaires were analysed. Forty-five percent of cardiologists were aware that diuretics can negatively affect sexual function, 93.1\% knew about the negative effects $\beta$-blockers can have, but only $9.2 \%$ were aware that ARBs can have
\end{abstract}

M. P. J. Nicolai $(\varangle) \cdot$ R. C. M. Pelger $\cdot H$. W. Elzevier Department of Urology, Leiden University Medical Center, PO box 9600, 2300, RC Leiden, the Netherlands e-mail: mpjnicolai@lumc.nl

\section{S. S. Liem}

Department of Cardiology, Amstelland Hospital, Amstelveen, the Netherlands

S. Both

Department of Psychosomatic Gynecology and Sexology,

Leiden University Medical Center, Leiden, the Netherlands

H. Putter

Department of Medical Statistics, Leiden University Medical Center, Leiden, the Netherlands

M. J. Schalij

Department of Cardiology, Leiden University Medical Center, Leiden, the Netherlands positive effects on sexual health. Almost half of respondents (48.2\%) stated they change medication regularly in an attempt to improve sexual function. Experienced cardiologists said they do this significantly more often than less experienced ones. Conclusions Cardiologists' knowledge about the effects of cardiovascular drugs on sexual health appears to be insufficient. Sexual dysfunction is not routinely taken into account when cardiologists prescribe drugs.

Keywords Sexualdysfunction · Adrenergic beta antagonists · Angiotensin-converting enzyme inhibitors · Angiotensine receptor antagonists · Diuretics · Calcium channel blockers . Hydroxymethylglutaryl-CoA reductase inhibitors

\section{Introduction}

Since the late 1980 s a rapid increase in the number of prescriptions for the treatment and prevention of cardiovascular disease (CVD) began.

The class of antihypertensive agents is the second most commonly prescribed class of medication, exceeded only by antidepressants [1]. In this time of high life expectancies, this therapy will be received during a substantial part of patients' lives [2]. The side effects induced by cardiovascular drugs are strongly associated with an impaired quality of life [3], affecting both patients and their partners [4]. Some classes of cardiovascular drugs have been described to cause sexual dysfunction. Diuretics and $\beta$-blockers can give rise to a decrease in libido, difficulty attaining or maintaining an erection and ejaculation problems [5-7]. However, recent literature describes beneficial effects on sexual health for thirdgeneration $\beta$-blockers [8], angiotensin II receptor blockers (ARBs) [9-12] and statins [13]. Existing data of experimental, observational and clinical studies consistently point towards similar effects of antihypertensive drugs on male and female 
sexual function [14-16]. As many as $70 \%$ of hypertensive patients who experience side effects are non-compliant with their antihypertensive medication and have a $40 \%-60 \%$ higher rate of therapy discontinuation, compared with patients whose quality of life is unaffected $[17,18]$. Since nonadherence with antihypertensive therapy can be life threatening, it is of great importance for cardiologists to be aware of the side effects that may be caused by the drugs they prescribe, including effects on patients' sexual function. Of course, cardiovascular drugs that improve symptoms and survival should not be withheld due to concerns about their potential impact on sexual function [19]. But whenever possible, multiple alternative options are available in order to provide patient-centred care. These options are described in part II of this article, which will be published in a later issue of this journal. Information about cardiologists' practice patterns concerning switches in medication, in order to improve patients' sexual function, is not yet available. Neither is it known whether cardiologists are aware of the different effects of cardiac drugs on sexual function. However, cardiologists will only be able to provide medicinal therapy with an optimum balance between efficacy and quality of life and to provide patients with sufficient information regarding the prescribed therapy if they have knowledge about these effects.

A recent report from our group revealed that Dutch cardiologists did not routinely discuss sexual function and a considerable number (41.9\%) of the respondents indicated a need for training to increase their knowledge to be able to discuss sexual matters with patients [20]. Accordingly, we hypothesised that information about the several effects of cardiovascular agents on sexual function has not yet permeated the cardiology practice. Therefore, aim of this study was to evaluate to what extent cardiologists are aware of the potential negative, neutral or beneficial effects that cardiovascular drugs can have on sexual function and whether this knowledge is actively used in patient care. We made use of additional data obtained during the National survey among cardiologists assessing their awareness, knowledge and practice patterns regarding sexual dysfunction in cardiac patients. Results of this survey, in combination with the review (part II), will provide cardiologists and residents in cardiology with the necessary information to be able to provide holistic healthcare.

\section{Methods}

Study design and sample

A national cross-sectional questionnaire survey was set among all active Dutch cardiologists and residents in cardiology (1054) who were members of the Netherlands Society of Cardiology (NVVC) in the autumn of 2011. However, some addresses from the society were out of date, leading to a total of 980 questionnaires which could be sent to the work addresses of NVVC members.

\section{Survey and procedure}

The study methodology was identical to a previously reported assessment of Dutch cardiologists, evaluating their inquiry into patients' sexual function [20]. The questionnaire was designed by the authors, based on a review of the literature. A multidisciplinary expert panel, with experience in developing surveys, checked the questionnaire for comprehensiveness and quality. The survey was pilot tested with 40 anonymous pilot questionnaires, which were distributed to cardiologists and residents in cardiology from the Leiden University Medical Centre. Of the pilot questionnaires, 23 were completed and returned (response rate $57.5 \%$ ). To improve the suitability and comprehensiveness of the questionnaire, it was adjusted according to the remarks made by the responding cardiologists. The questionnaire comprised 31 questions focusing on current practice in addressing sexual health and knowledge about (side) effects of the eight main classes of cardiovascular drugs. Questionnaires were accompanied by a letter explaining the nature, scope, objectives and contents of the questionnaire and total anonymity was ensured. A free post return envelope was added, as well as an opt-out possibility. Cardiologists not interested in participation could answer a question regarding the reason(s) not to participate and return the questionnaire empty. Reminder letters were sent to non-respondents 2, 5 and 7 months after the initial mailing.

Results about cardiologists daily practice patterns regarding assessment of sexual function were published separately [20].

\section{Data analysis}

Means of numerical demographic values and answers to the questions were analysed with frequencies. Bivariate associations between demographic information and the categorical data were calculated using the Pearson chi-square procedure, for ordinal variables Armitages' trend test was used. Associations between numerical data and demographics of the respondents were analysed with the independent sample t-tests. Twosided $P$ values $<0.05$ were considered statistically significant. Some questions with more than one possible answer and with open answers were grouped together for analysis. Data were analysed by SPSS release 20 (SPSS Inc., Chicago, IL, USA).

\section{Results}

\section{Demographics}

Of the 980 eligible participants, 528 surveys were returned, yielding an unweighted response rate of $53.9 \%$. Seven 
participants were eliminated because they were not participating in a clinical setting or because they were physician assistants. A total of 98 questionnaires were eliminated because they were empty or incomplete for more than $60 \%$ and 16 because they lacked answers to the most important questions. Reasons mentioned for the inability to fill in the questionnaire $(n=114)$ were: not interested $(n=4)$, no time $(n=2)$, or not enough experience in the field $(n=2)$. Others indicated they were only working with specific patient groups such as children or pregnant women and therefore were not able to fill in the questionnaire $(n=5)$. However, most cardiologists who indicated unwilling to participate, gave no reason for this $(n=101)$. In total $78.4 \%$ of the returned surveys were analysed $(n=414)$. Mean age of the respondents was 45.5 years $( \pm 9.60)$, $76.0 \%$ were male, $80.9 \%$ were cardiologists and $19.1 \%$ were residents. Female respondents were significantly younger than male respondents (mean $42.3 \pm 8.37$ and $46.6 \pm 9.75$, respectively; $p<0.001$ ), consequently more female respondents were residents $(p<0.001)$. Demographic characteristics of nonrespondents were not available for analysis due to the anonymous design of the survey. The personal and practice characteristics of respondents are summarised in Table 1.

Knowledge about sexual side effects

More than one fourth $(27.5 \%)$ of respondents stated they have 'not much' of the knowledge necessary to discuss sexual

Table 1 Respondent characteristics $(n=414)$

\begin{tabular}{lc}
\hline Age (years) & $\mathrm{n}(\%)$ \\
$\leq 45$ & $208(50.2)$ \\
$>45$ & $206(49.8)$ \\
Sex & $\mathrm{n} *(\%)$ \\
Male & $314(76.0)$ \\
Female & $99(24.0)$ \\
Function & $\mathrm{n}(\%)$ \\
Cardiologist & $335(80.9)$ \\
Resident & $79(19.1)$ \\
Type of clinic/practice & $\mathrm{n} *(\%)$ \\
Tertiary referral centre (or university hospital) & $90(21.8)$ \\
District general teaching hospital & $174(42.2)$ \\
District general hospital & $148(35.9)$ \\
Time of practice in cardiology & $\mathrm{n} *(\%)$ \\
$0-11$ month & $18(4.3)$ \\
$1-2$ years & $30(7.2)$ \\
3-5 years & $80(19.3)$ \\
$6-10$ years & $106(25.6)$ \\
$11-15$ years & $49(11.8)$ \\
15 years or more & $131(31.6)$ \\
\hline
\end{tabular}

* $\mathrm{n}$ differs because the questions were not answered consistently, some were skipped or forgotten function with patients, $2.0 \%$ said they have no knowledge about this at all. The majority of cardiologists reported having 'some' $(65.1 \%)$ and only $5.4 \%$ 'a lot' of the necessary knowledge to discuss sexual problems with patients. Respondents were asked to note which effect on sexual function they thought was caused by each class of cardiovascular drugs (positive, neutral or negative). For $\beta$-blockers, $93.1 \%$ of the cardiologists believed these to have negative effects on sexual function. Significantly more female physicians thought $\beta$ blockers have a positive effect on sexual function compared with male physicians $(9.1 \%$ and $1.6 \%$, respectively; $p<$ 0.001). Regarding diuretics, respondents gave more diverse answers: $57.7 \%$ said they have a neutral effect and $40.5 \%$ knew they have a negative effect on sexual function. The majority $(64 \%)$ indicated ARBs to have a neutral effect on sexual function, only $9.2 \%$ knew this class can have beneficial effects on sexual function. Cardiologists' answers regarding the presumed effects of cardiovascular drugs on sexual function are illustrated in Fig. 1. No significant differences between male and female physicians were seen.

Counselling about sexual side effects

The majority of the responding cardiologists $(76.6 \%)$ stated to counsel their patients regularly or often about sexual side effects of cardiovascular drugs. Fifteen percent said they inquired about sexual problems when a patient had recently started using cardiac medication and $74.4 \%$ when a patient recently started using a $\beta$-blocker. On the contrary, only $19.3 \%$ of respondents inquired regularly or often about PDE-5 inhibitor use, while $43.6 \%$ said they informed patients

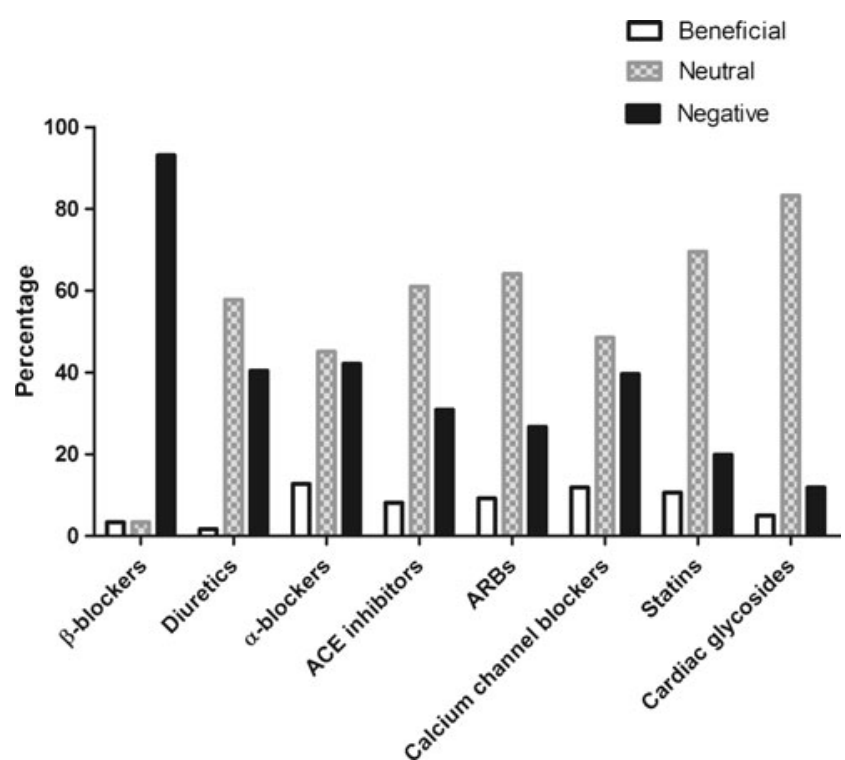

Fig. 1 Effects of eight classes of cardiovascular medication on the sexual function as presumed by the responding cardiologists, shown in percentages. Based on answers of at least 400 cardiologists (in training) per category 
about the possibility to use a PDE-5 inhibitor after a myocardial infarction (MI) or in patients with heart failure. Furthermore, $48.2 \%$ of respondents said they changed medications regularly or often in an attempt to improve sexual function (Table 2). Significant differences were seen regarding this statement; cardiologists stated to switch significantly more often than residents $(p<0.001)$. Finally, the more knowledge about sexual function cardiologists thought they had, the more often they changed medication in an attempt to improve sexual function (linear-by-linear association, $p=0.002$ ) and the more often they counselled patients about effects and use of erection potentiating medication (linear-by-linear association, $p=0.032$ and $p<0.001$, respectively).

\section{Discussion}

This study indicates cardiologists are not fully aware of the effects cardiovascular drugs can have on sexual function. Although the majority of responding cardiologists stated they informed patients about sexual side effects from the medication they prescribe, their knowledge of these effects seems incomplete. Most cardiologists designated negative effects on sexual function to $\beta$-blockers, although third-generation $\beta-1$ blockers have been shown to have beneficial effects on sexual function. Furthermore, the lion's share of respondents thought no effects on sexual function were to be expected from most other classes of cardiovascular drugs, underestimating that most classes of cardiovascular drugs either have a beneficial or a negative effect on sexual function. Interestingly, more experience as a cardiologist was positively associated with informing about sexual function and counselling about side effects, suggesting that more education about sexual health in cardiologists' training could improve awareness and thus quality of patient care on this point.

This was the first survey among cardiologists assessing their knowledge about (side) effects of cardiovascular agents and their practice patterns regarding switching of medication in order to resolve or avoid sexual dysfunction. The findings should be considered in the light of potential study limitations. First, the self-reported character may have led to response and selection bias although the response rate was sufficient and the demographics found were comparable with the national figures. Second, we made use of a non-validated questionnaire, since validated questionnaires about this subject are not available. Third, the questionnaire was designed to obtain information about cardiologists' knowledge and practice pattern concerning both male and female sexual function. Most trials investigating effects of cardiovascular drugs on sexual function are specifically designed to measure effects on erectile function since this is a common problem which is easy to measure with one of the available validated questionnaires [21]. And, because erectile dysfunction has been shown to be a sentinel marker for risk of cardiovascular disease [22] it has received full attention in the past years, while very little attention was paid to female sexual dysfunction. Respondents may therefore answered the questionnaire with erectile dysfunction in mind, this may have led to an upward distortion of the results.

Our data indicate that there is room for improvement regarding cardiologists' knowledge about the sexual (side) effects of cardiovascular drugs. To improve compliance of therapy in cardiology, patients should be properly informed about side effects that can be expected from a drug and equally important, patients should have the possibility to discuss a switch in medication with their physician when sexual dysfunction occurs. Large clinical trials about the effects of switching are lacking and should be conducted in the near future. However, available data from observational studies and clinical trials point consistently towards significant benefits for sexual function when prior antihypertensive therapy is switched to either nebivolol or an ARB (Part II and references 5 and 23) as significant improvements were observed in orgasmic function and intercourse satisfaction, along with benefits in sexual desire and frequency of sexual intercourse $[11,23]$. From an different angle, the American Heart Guideline [24] states that sexual dysfunction has not been associated with the use of ACE inhibitors, ARBs and calcium channel blockers. Cardiologists should be aware that these drug classes can be prescribed safely.

Obviously, patients' health and cardiac condition take precedence above sexual function. In young patients with chronic

Table 2 Counselling and informing patients with erectile dysfunction

\begin{tabular}{|c|c|c|c|c|c|}
\hline $\begin{array}{l}\text { How often do you counsel patients regarding erectile } \\
\text { function (EF) after MI or with heart failure about: }\end{array}$ & Never n $(\%)$ & Seldom n $(\%)$ & Regularly n (\%) & Often n $(\%)$ & $\mathrm{n}^{*}$ (total) \\
\hline Use of nitrates with pain & $42(10.4)$ & $112(27.8)$ & $145(36.0)$ & $104(25.8)$ & 403 \\
\hline Effects of medication on EF & $15(3.7)$ & $80(19.8)$ & $197(47.5)$ & $110(27.2)$ & 404 \\
\hline Use of PDE5 inhibitors & $27(6.7)$ & $202(49.8)$ & $154(37.9)$ & $23(5.7)$ & 406 \\
\hline How often do you: & Never n (\%) & Seldom n $(\%)$ & Regularly n (\%) & Often n $(\%)$ & $\mathrm{n} *$ (total) \\
\hline Inform about erection potentiating agents & $93(23.6)$ & $234(57.6)$ & $59(14.5)$ & $20(4.9)$ & 406 \\
\hline Change medication to improve sexual function & $17(4.2)$ & $193(47.7)$ & $176(43.5)$ & $19(4.7)$ & 405 \\
\hline
\end{tabular}

* $\mathrm{N}$ differs because the questions were not answered consistently, some were skipped or forgotten. 
heart failure, for example, especially those awaiting heart transplantation, the use of $\beta$-blockers is of the utmost importance to improve survival and should not be withheld in order to preserve sexual function. In addition, without even taking the comorbidities into account, heart failure itself often leads to deterioration in the physical condition and to erectile dysfunction independent of the medication used [25]. Choices for medicinal treatment should always be balanced between cardiologists' expert judgments and patients' wishes. But while guidelines highly recommend routine assessment of sexual function before initiation of antihypertensive treatment [26], our results showed that inquiry about sexual function is not yet routine in cardiology practice. A significant number of Dutch cardiologists lacked the knowledge to be able to provide accurate information about the effects cardiovascular agents can have on sexual function. Experience and sufficient selfperceived knowledge about sexual health appeared to be crucial factors for inquiring and dealing with medicinal effects on sexual function. Therefore, education about these (side) effects in cardiologists training could be useful to enable cardiologists to deal with patients presenting with sexual dysfunction during treatment with cardiovascular agents.

Acknowledgements We would like to thank Prof. T. Jaarsma at the University of Linköping, Sweden and Tialda Hoekstra from the University Medical Center Groningen, the Netherlands. We partly based our questionnaire on a survey of their design.

Furthermore we thank Joyce van Bavel for helping us with the import of the data

Data sharing statement Additional unpublished data comprising cardiologists' knowledge and practice patterns regarding sexual dysfunction are in the possession of our research group. Because of the extensiveness of the subject, these data will be published in a separate paper.

Funding No external funding.

Conflict of interests None declared.

Open Access This article is distributed under the terms of the Creative Commons Attribution License which permits any use, distribution, and reproduction in any medium, provided the original author(s) and the source are credited.

\section{References}

1. Cherry DK, Woodwell DA, Rechtsteiner EA. National Ambulatory Medical Care Survey: 2005 summary. Adv Data. 2007;387:1-39.

2. Burchardt M, Burchardt T, Baer L, et al. Hypertension is associated with severe erectile dysfunction. J Urol. 2000;164(4):1188-91.

3. Wassertheil-Smoller S, Blaufox MD, et al. Effect of antihypertensives on sexual function and quality of life: the TAIM Study. Ann Intern Med. 1991;114(8):613-20.

4. Corona G, Petrone L, Mannucci E, et al. Assessment of the relational factor in male patients consulting for sexual dysfunction: the concept of couple sexual dysfunction. J Androl. 2006;27(6):795-801.
5. Dusing R. Sexual dysfunction in male patients with hypertension: influence of antihypertensive drugs. Drugs. 2005;65(6):773-86.

6. Fogari R, Preti P, Derosa G, et al. Effect of antihypertensive treatment with valsartan or atenolol on sexual activity and plasma testosterone in hypertensive men. Eur J Clin Pharmacol. 2002;58(3):177-80.

7. Baumhakel M, Schlimmer N, Kratz M, Hackett G, Jackson G, Bohm M. Cardiovascular risk, drugs and erectile function-a systematic analysis. Int J Clin Pract. 2011;65(3):289-98.

8. Brixius K, Middeke M, Lichtenthal A, Jahn E, Schwinger RH. Nitric oxide, erectile dysfunction and beta-blocker treatment (MR NOED study): benefit of nebivolol versus metoprolol in hypertensive men. Clin Exp Pharmacol Physiol. 2007;34(4):327-31.

9. Chen Y, Cui S, Lin H, et al. Losartan improves erectile dysfunction in diabetic patients: a clinical trial. Int J Impot Res. 2012;24(6):217-20.

10. Dusing R. Effect of the angiotensin II antagonist valsartan on sexual function in hypertensive men. Blood Press Suppl. 2003;2:29-34.

11. Manolis A, Doumas M. Antihypertensive treatment and sexual dysfunction. Curr Hypertens Rep. 2012;14(4):285-92.

12. Pauls RN, Kleeman SD, Segal JL, Silva WA, Goldenhar LM, Karram MM. Practice patterns of physician members of the American Urogynecologic Society regarding female sexual dysfunction: results of a national survey. Int Urogynecol J Pelvic Floor Dysfunct. 2005;16(6):460-7.

13. Trivedi D, Kirby M, Wellsted DM, et al. Can simvastatin improve erectile function and health-related quality of life in men aged $>/=40$ years with erectile dysfunction? Results of the Erectile Dysfunction and Statins Trial [ISRCTN66772971]. BJU Int 2012 Jun 11.

14. Ma R, Yu J, Xu D, et al. Effect of felodipine with irbesartan or metoprolol on sexual function and oxidative stress in women with essential hypertension. J Hypertens. 2012;30(1):210-6.

15. Doumas M, Tsiodras S, Tsakiris A, et al. Female sexual dysfunction in essential hypertension: a common problem being uncovered. J Hypertens. 2006;24(12):2387-92.

16. Fogari R, Preti P, Zoppi A, et al. Effect of valsartan and atenolol on sexual behavior in hypertensive postmenopausal women. Am J Hypertens. 2004;17(1):77-81.

17. Ferrario CM, Levy P. Sexual dysfunction in patients with hypertension: implications for therapy. J Clin Hypertens (Greenwich). 2002;4(6):424-32.

18. Croog SH, Levine S, Testa MA, et al. The effects of antihypertensive therapy on the quality of life. N Engl J Med. 1986;314(26):1657-64.

19. Levine GN, Steinke EE, Bakaeen FG, et al. Sexual Activity and Cardiovascular Disease: A Scientific Statement From the American Heart Association. Circulation. 2012;125(8):1058-72.

20. Nicolai MP, Both S, Liem SS, et al. Discussing sexual function in the cardiology practice. Clin Res Cardiol 2013 Feb 8.

21. Rosen RC, Riley A, Wagner G, Osterloh IH, Kirkpatrick J, Mishra A. The international index of erectile function (IIEF): a multidimensional scale for assessment of erectile dysfunction. Urology. 1997;49(6): 822-30.

22. Dong JY, Zhang YH, Qin LQ. Erectile dysfunction and risk of cardiovascular disease: meta-analysis of prospective cohort studies. J Am Coll Cardiol. 2011;58(13):1378-85.

23. Baumhakel M, Schlimmer N, Bohm M. Effect of irbesartan on erectile function in patients with hypertension and metabolic syndrome. Int J Impot Res. 2008;20(5):493-500.

24. U.S. Department of Health and Human Services NHLaBINioH. The seventh report of the Joint National Committee on Prevention, Detection, Evaluation, and treatment of High Blood Pressure. Complete report. 2004.

25. Hoekstra T, Jaarsma T, Sanderman R, van Veldhuisen DJ, LesmanLeegte I. Perceived sexual difficulties and associated factors in patients with heart failure. Am Heart J. 2012;163(2):246-51.

26. Hackett G, Kell P, Ralph D, et al. British Society for Sexual Medicine guidelines on the management of erectile dysfunction. J Sex Med. 2008;5(8):1841-65. 